\title{
Problem restructuring processes for ill-structured verbal analogies
}

\author{
G. MICHAEL BARNES and SUSAN E. WHITELY \\ University of Kansas, Lawrence, Kansas 66045
}

\begin{abstract}
The processes involved in analogy solving have been an important investigative area in cognitive psychology. Although problem restructuring has been a central construct in problem solving theory, no restructuring processes have been proposed for analogical reasoning. Yet, the stimulus terms for analogies, as they appear on ability tests, are often ill-structured. That is, they are ordered in a way that does not permit direct problem comprehension. In the current study, both perceptual and semantic problem restructuring processes were hypothesized for analogy solving. The independence, stage of execution, and susceptibility to strategic control of the two processes were examined. The results from two experiments indicated that (1) ill-structured analogies are restructured during problem solving, (2) perceptual and semantic restructuring processes are independent and executed at different stages of analogy solving, and (3) both processes exhibited automaticity since repetition of analogy solution attenuated but did not eliminate either restructuring process. A model of analogical reasoning that incorporated both restructuring processes and their execution sequences was proposed. The nature and automaticity of perceptual and semantic analogy restructuring processes were disscussed.
\end{abstract}

Verbal analogies have been an intriguing problem solving task in cognitive psychology research. This is indicated by the several models of problem solving processes for analogies that have been examined (Pellegrino \& Glaser, 1980; Reitman, 1965; Rumelhart \& Abrahamson, 1973; Spearman, 1923; Sternberg, 1977a; Whitely \& Barnes, 1979). Several features of analogies contribute to their popularity for study. First, verbal analogies are semantically rich problems requiring the education, production, and evaluation of word pair relationships. Second, verbal analogies are reasonably well-structured problems that are suited to stage analysis because the solution must fulfill a set of specified constraints. These constraints include the size of the alternative set and a restricted set of possible semantic relationships (Whitely, 1977). Third, since analogy items have the greatest saturation on the general intelligence factor that is common among intelligence tests (McNemar, 1964), they can be used to model individual differences from information processing components of human intelligence. An example of such an attempt is Sternberg's (1977a) componential theory of analogical reasoning. Fourth, modeling procedural aspects of human intelligence also has heuristic impact for artificial intelligence (Evans, 1968; Hayes-Roth \& McDermott, 1978; Reitman, 1965).

This research was partially supported by National Institute of Education Grant NIE-6-7-0156 to S. E. Whitely and represents part of G. M. Barnes' doctoral dissertation. G. M. Barnes is now at the Computer Science Department, Kansas State University, Manhattan, Kansas 66506 . The authors would like to thank Kristin J. Bruno for her suggestions on the manuscript.
Consider the prototypic structure of an analogy:

$$
\begin{aligned}
& \mathrm{A}: \mathrm{B}:: \mathrm{C}: \stackrel{?}{-} \quad \text { [stem] } \\
& \begin{array}{lllll}
\mathrm{X} 1 & \mathrm{X} 2 & \mathrm{X} 3 & \mathrm{X} 4 & \text { [alternatives] }
\end{array}
\end{aligned}
$$

The order of the elements in the stem conveys implicit problem-defining information. The analogy problem is read. " $A$ is to $B$ as $C$ is to some unknown. What alternative has the same relationship with $\mathrm{C}$ as $\mathrm{A}$ has with B?"

Sternberg (1977a, 1977b) has proposed a detailed model that incorporates six information processing components: encoding, inference, mapping, application, justification, and preparation-response. Two of these processes, preparation-response and encoding, concern relationships between the physical stimulus and an internal representation of the problem. Preparationresponse is a motor process that represents the solver's orientation to the problem and the response output. Encoding is the construction of an internal representation of the analogy terms from the physical stimuli. The remaining processes elaborate, or modify, the current problem representation. Inference and mapping processes determine the $\mathrm{A}: \mathrm{B}$ and $\mathrm{A}: \mathrm{C}$ stem relations, respectively. Application uses the mapping relationship to extend the domain (inference) of the analogy to its range ( $\mathrm{C}$ term and alternatives) and determines possible answers. Justification, an optional process, is used to decide among competing alternatives, if necessary. Encoding and preparation-response are exhaustively executed. The other processes may be executed in either a self-terminating or an exhaustive fashion. The initialization sequence of process execution is: preparation, 
encoding, inference, mapping, application, justification, and response. With the exception of mapping, similar processes have been incorporated in previous analogical models (Evans, 1968; Johnson, 1962; Reitman, 1965; Rumelhart \& Abrahamson, 1973; Spearman, 1923).

Current models of analogical reasoning either assume that analogies are well-structured problems or ignore the issue. None of the previously mentioned models is directly applicable to ill-structured verbal analogies because they do not incorporate analogy restructuring processes. Ill-structured verbal analogies, however, often occur in aptitude measurement instruments and among some experimental analogy sets. For example, solve the following analogy, which is like many psychometric analogies:

\section{ROBIN : MUSTANG :: BIRD : — ? \\ LIZARD HORSE DOG FISH}

Clearly, this is a poorly structured version of the following analogy:

\section{ROBIN : BIRD :: MUSTANG : _ _ ? \\ LIZARD HORSE DOG FISH}

The well-structured domain and range of the example are: Specific Animal 1 (ROBIN) "is a member of" Animal Category 1 (BIRD) as Specific Animal 2 (MUSTANG) "is a member of" Animal Category 2 (_? - ). The ill-structured domain and range are: Specific Animal 1 (ROBIN) "is an animal name as is" Specific Animal 2 (MUSTANG) as Animal Category 1 (BIRD) "is an animal category name as is" Animal Category 2 (_ ? - ). The domain of the ill-structured example cannot produce a selection rule between the alternatives; they are all animal categories.

Although Sternberg (1977b, pp. 232-233) briefly addressed the issue of analogy ill-structure, he was unable to support the inclusion of a restructuring process in his componential model. Sternberg tested a variant model that utilized the strongest stem word pair relationship (either $\mathrm{A}: \mathrm{B}$ or $\mathrm{A}: \mathrm{C}$ ) as the analogy domain and the weakest relationship as the mapping component. He was unable to obtain substantial differences between his preferred model and the variant model. It should also be noted that Sternberg presented a mix of ill-structured and well-structured analogies, but he did not compare both versions of the same analogies. Grudin (1980) has observed that several of Sternberg's ill-structured analogies were solved faster when presented as well-structured, prototypic analogies. Grudin concluded that the $A: C$ word pair relation was utilized as the domain when the $\mathrm{A}: \mathrm{B}$ word pair relation was not applicable. However, the mapping component is not to be construed as a problem restructuring process. Barnes (Note 1) has obtained mapping latencies for true-false, but not for forced-choice, well-structured verbal analogies.

If models of analogical reasoning are to be used to understand intelligence and link individual difference research with experimental cognitive research, then problem solving for well-structured and ill-structured analogies must be modeled. To accomplish this goal, models of analogical reasoning must postulate and investigate the role of problem restructuring processes. The purpose of the following discussion and two experiments is to propose and examine the nature of two problem restructuring processes for analogies. Specific emphasis will be placed on their independence, stages of execution, and susceptibility to strategic control.

\section{Problem Restructuring Processes}

The restructuring of ill-structured problems has been an enduring and central construct in psychological models of human problem solving. Problem restructuring concepts and mechanisms have been espoused by the Gestaltist (Duncker, 1945; Luchins, 1942), the associationist (Maltzman, 1955; Mednick, 1962), and the contemporary cognitive psychologist (Greeno, 1976; Newell \& Simon, 1972; Simon, 1973) traditions. Problem restructuring is a necessary construct because it postulates that the internal representation of problems can be changed in the course of problem solving to achieve solution. Generally, a problem is considered well-structured if all relevant information concerning the problem, the strategy needed, and the attributes of a successful solution are known by the solver. For wellstructured problems, the steps to solution are usually well-known and solution is primarily an algorithmic, or reproductive, sequence of problem solving processes (Greeno, 1973). Well-structured problems can be typified by protocols for solution strategy (Greeno, 1976; Newell \& Simon, 1972; Simon \& Reed, 1976). These protocols usually represent a strategy of working forward from the initial state to a familiar goal state. IIIstructured problems, on the other hand, do not have an explicit statement of the task environment. Likewise, the steps on the solution path and the attributes of a correct solution are not completely known as problem solving begins (Davis, 1973; Greeno, 1973; Simon, 1973). Ill-structured problems are often solved by working backward from the goal to the current problem state.

Although problem restructuring is a central construct in problem solving models, little experimental research has been devoted to understanding problem restructuring as an information processing component. That is, problem restructuring processes have not been studied by comparing solution latencies for well-structured and ill-structured versions of the same problem. Rather, current problem solving research has (1) developed taxonomies of problem structures and problem solving processes (e.g., Davis, 1973; Greeno, 1973; Simon, 
1973), (2) used protocol and means-ends analyses to develop information processing models of general problem solvers and strategies for solving problems (e.g., Newell \& Simon, 1972; Simon \& Reed, 1976), and (3) developed stage models for rapidly solved verbal problems (e.g., Clark \& Chase, 1972; Potts \& Scholz, 1975; Sternberg, 1979). Similarly, research on verbal analogies has concerned a comparable range of topics, but, as yet, analogy restructuring processes have not been proposed or experimentally studied.

\section{Well-Structured and IIl-Structured Verbal Analogies}

Given the prototypic analogy structure and two transformational rules, three ill-structured versions of an analogy can be generated. The first transformation, structural, exchanges the $B$ and the $C$ terms in the analogy stem. The second transformation, directional, changes the direction of the analogy stem.

Consider the following four versions of the same verbal analogy. The first problem is a prototypic, wellstructured analogy:

\section{$[\mathrm{A}: \mathrm{B}: \mathrm{C}:-]$ \\ DEEP : SHALLOW : : CHEAP : __ \\ COSTLY, WIDE}

Application of the structural transformation produces a semantically ill-structured version of the analogy. In this version, the inference, mapping, and application components of stem representation can be easily confused:

$$
[\mathrm{A}: \mathrm{C}: \mathrm{B}:-]
$$

DEEP : CHEAP :: SHALLOW : __?

\section{COSTLY, WIDE}

A perceptually ill-structured version of the analogy can be produced by applying the directional transformation to the prototypical analogy. In this version, the direction in which the analogy problem is initially encoded and internally represented is the reverse of the order of the relationships in the analogy stem:

\section{$\left[\_: \mathrm{C}: \mathrm{B}: \mathrm{A}\right]-?-:$ CHEAP $::$ SHALLOW : DEEP \\ COSTLY, WIDE}

The last ill-structured version is obtained by applying both structural and directional transformations to the prototypic analogy. The result is an analogy in which the direction of the initial stem encoding and the stem relationship eduction processes are poorly structured and possibly misunderstood:

\section{$[-: B:: C: A]$ \\ $?$ ? : SHALLOW : : CHEAP : DEEP \\ COSTLY, WIDE}

The four versions of verbal analogy structure can be conceptualized as a fourfold table. The two dimensions represent perceptual and semantic analogy structure. Both dimensions have two values for analogy structures that do, or do not, require analogy restructuring, for example:

\begin{tabular}{ccc} 
Semantically & \multicolumn{2}{c}{ Perceptually Restructure } \\
\cline { 2 - 3 } Restructure & \multicolumn{1}{c}{ No } & Yes \\
\hline No & A :B::C:- & $-: C:: B: A$ \\
Yes & A :C::B:- & $-: B:: C: A$
\end{tabular}

Clearly, the above four verbal analogy structures are a subset of all possible ill-structured versions. They are, however, common forms of ill-structured verbal analogy stems. Thus, analogical restructuring processes applicable to the above three versions of analogy ill-structure should generalize to some degree to other versions of ill-structured analogies.

\section{EXPERIMENT 1}

The first experiment examined the independence, susceptibility to strategic control, and stage of execution for both perceptual and semantic analogy restructuring processes. Solution latencies and accuracy for the four structural versions of analogy problems were compared. In addition, each analogy in one of the four analogy structures was solved three times.

Susceptibility to strategic control. Repetition was included in the design to determine if analogy restructuring is either susceptible to strategic control or automatically executed. With repetitive problem solving, subjects could adopt a solution recognition strategy and thus circumvent analogy restructuring processes. They would merely have to pair solutions with analogies regardless of the analogy's structure. Then, upon recognition of the analogy, solution could be achieved by the simpler process of cued recall.

Repetitive problem solving has been shown to reduce steps to solution (Simon \& Reed, 1976). Thus repetitive problem solving was expected to make the problems more well-structured, since the solver presumably knew more about the necessary steps to achieve solution. Therefore, repetition should decrease solution latency and increase accuracy.

The elimination of analogy restructuring with increasing problem solving trials would suggest a strategy shift from problem solving to solution recognition. A failure to eliminate analogy restructuring effects by repetition would suggest that subsequent analogy restructuring processes are performed automatically. That is, analogy restructuring processes are always executed and are not subject to strategic control. The facilitation of analogy solving without the elimination of analogy restructuring effects with repetition would indicate a shift from productive to algorithmic problem solving. That is, with additional solution trials, the correct sequence of problem solving processes becomes learned, the solution 
known, and analogy solving facilitated, although all processes are still performed.

Stage of execution. Three analogical problem tasks were used to determine the stage of execution for both analogy restructuring processes. One analogy task presented an intact verbal analogy and required subjects to solve the problem. The other two problem tasks factored the analogy problem into an analogy stem task and an analogy solution task. The analogy stem task presented the analogy stem for study. The analogy solution task followed the analogy stem task and presented the alternatives with the stem for the analogy problem. The analogy was solved during the analogy solution task.

Potts and Scholz (1975) used a similar set of tasks to examine the effects of task presentation on the internal representation for three-term series problems. They noted different internal representations for the three-term series problems if the problem question was presented simultaneously with the problem's premise (an intact task) or was presented separately (the decomposed task). The three problem tasks were used to examine the role the alternative set has on analogy restructuring. The analogy stem and analogy solution tasks separated the internal representation of the analogy's premise (stem) in the stem task from the production and evaluation of the solution in the analogy solution task. The analogy stem and solution tasks were similar to Sternberg's (1977a, 1977b) three-cue precuing and solution intervals, respectively. The intact task is comparable to Sternberg's solution interval for the zero-cue precuing condition.

Restructuring hypotheses. The four structural versions of analogy problems were used to examine three analogy restructuring hypotheses. The first hypothesis was that response latencies attributable to semantic and perceptual analogy restructuring transformations could be obtained. Increased response latency for illstructured analogies would support problem restructuring components in analogical reasoning.

The second hypothesis was that the semantic and perceptual restructuring transformations represent separate processing stages. The latency, stage of execution, and accuracy for both restructuring processes were examined to test this hypothesis. Significant main effects for the latencies of both restructuring processes without a significant interaction would support their independence. Alternatively, an interaction would support a more general, non-transformation-specific restructuring stage. In addition, the latencies for both types of restructuring processes were compared by contrasting the $\mathrm{A}: \mathrm{C}:: \mathrm{B}:$ and $_{-}$and the $\mathrm{C}: \mathrm{B}: \mathrm{A}_{\text {response }}$ times.

Both restructuring processes were predicted to occur at different times in the course of problem solving and to have differential effects on solution accuracy. Perceptual ill-structure was hypothesized to be immediately apparent, whereas semantic ill-structure was thought to be less discernible. The _ $: \mathrm{C}:: \mathrm{B}: \mathrm{A}_{\text {and }}$ the $\_: \mathrm{B}:: \mathrm{C}: \mathrm{A}$ ill-structured analogies provide a perceptual trigger for analogy restructuring in the stem-blank position. When analogy solvers encode a blank as the first stem position, they should be aware that the stem relationships have been reversed. Perceptual analogy restructuring was expected to manifest effects in the analogy stem task but not in the analogy solution task due to the stem-blank position. The $\mathrm{A}: \mathrm{C}:: \mathrm{B}:{ }_{-}$and the $\_: \mathrm{B}:: \mathrm{C}: \mathrm{A}$ analogy structures, on the other hand, do not provide a salient cue for semantic problem restructuring. Semantic ill-structure within the stem components can be determined only by attempts to educe relationships. Apprehension of stem ill-structure is further confounded by the nature of the inference, mapping, and application components. That is, the need to semantically restructure can become apparent during stem processing, but it is more likely to be perceived as a result of unsuccessful application processing. Thus, semantic analogy restructuring was expected to manifest effects in both the analogy stem and solution tasks. Since semantic restructuring requires relationship eduction, failure to semantically restructure an ill-structured analogy was expected to decrease solution accuracy. Conversely, perceptual analogy restructuring should be independent of accuracy due to its obvious perceptual trigger.

The third hypothesis was that both the Perceptual Restructuring by Repetition and the Semantic Restructuring by Repetition interactions would be significant. There were three possible interpretations of the two interactions. First, significant Restructuring by Repetition interactions, facilitation of analogy solving across repetition, and significant restructuring effects at all levels of repetition would indicate that both restructuring processes were automatic and executed more efficiently (algorithmically) with repetition. Second, an interaction that results in the elimination of analogy restructuring effects across repetition would indicate a shift to a strategy in which restructuring need not occur (e.g., solution recognition). Third, no significant Restructuring by Repetition interactions, with significant main effects for both restructuring processes, would indicate that the restructuring processes were automatically executed and were not facilitated by repetitive analogy solving.

\section{Method}

Subjects and Apparatus. Twenty-four subjects from a large state university in the Midwest participated in the experiment as a requirement of an introductory psychology course. The experimental stimuli were presented by slide projector. The projector light triggered a millisecond timer that was terminated by subject response. Responses and latencies were printed on paper tape as the experiment progressed. Individual subjects were tested in a 1-h session. Each subject responded to 64 experimental analogies. This corresponded to eight analogies for each experimental condition. The experimental analogies came from an item bank calibrated by Whitely and Dawis (1976) to fit a test theory model. Forced-choice verbal analogies with 
two alternatives, as in the previous examples, were used. The correct answer was the first (left) or second (right) alternative half of the time for each position. Subjects solved the problem by throwing the response switch in the same direction as their chosen solution. If their chosen solution was the second alternative, they threw the switch to the right.

Design. The experimental design was a 2 (intact or decomposed task) by 2 (perceptual structure) by 2 (semantic structure) by 3 (repetition of problem solving) within-subjects factorial. The three analogical problem tasks were analyzed separately. The two dependent variables, response time and response accuracy, were expected to be negatively correlated. To control for an expected speed-accuracy tradeoff, response latency was analyzed with accuracy as a covariate. Accuracy was analyzed in a separate univariate case.

The order of the intact analogy and the decomposed analogy tasks (the analogy stem and solution tasks) was counterbalanced across subjects. Eight experimental analogies were arbitrarily assigned to one of eight groups, for a total of 64 analogies. Thus, there were eight problem solving trials (replications) for each experimental condition. The eight experimental conditions were counterbalanced across subjects. Each group of eight analogies was presented in every condition of analogy structure and analogy problem task three times in the total data set. However, each subject solved the same set of experimental analogies in the same experimental conditions three times. All three analogy tasks were solved prior to the second repetition interval.

Procedure. Subjects read an instruction sheet explaining the experiment as an investigation of the time it takes to represent and solve analogy problems. Analogies were explained with examples. The response options were explained to the subjects, along with a description of an experimental trial. Subjects responded to 16 practice trials prior to each of the initial problem tasks.

For the analogy stem task, subjects were told to terminate presentation of the stem when they understood the problem by initiating a right response. The subject's response initiated the analogy solution task (presentation of the stem and alternatives). The analogy solution task was terminated when the subject solved the problem. The subject's response to the intact analogy problem task terminated presentation of the problem. Subjects were able to initiate every trial by responding to a filler slide with "left" or "right" typed on it. This slide presented the correct response for the preceding analogy. Thus, subjects could monitor and adjust their ongoing speed-accuracy criterion. Subjects were instructed to respond as rapidly as possible while making few errors.

\section{Results}

The two dependent variables were response time and a $\log$ accuracy measure, $\operatorname{Ln}[\mathrm{P}($ correct $) / \mathrm{P}$ (error) $]$. As recommended by Pachella (1974), the log accuracy transformation was used to yield a more linear speedaccuracy tradeoff. Geometric means were used to estimate a response time and an accuracy measure from the eight replications of each experimental condition. The distributions of response time and accuracy estimated by geometric means were slightly less skewed and more mesokurtotic than the corresponding arithmetic mean distributions. These estimates of response time and accuracy were the units of analysis.

Response latency and accuracy were negatively correlated over subjects for the three analogy problem tasks. The respective tradeoff correlations for the intact analogy, analogy stem, and solution tasks were: $\mathrm{r}=-.11, \mathrm{r}=-.13$, and $\mathrm{r}=-.13$, with all probabilities $<.03(n=288)$. Thus response latency and accuracy were slightly related. Tables 1 and 2 depict the latency and accuracy means for perceptual and semantic restructuring at each interval of repetition. Since the analogy stem task and solution task are portions of the same analogy problem, their accuracy is assessed only in the solution task. The average percent correct for the intact analogy task and the decomposed analogy tasks were $88 \%$ and $89 \%$, respectively.

Table 1

Response Latency Means (in Milliseconds Adjusted for Accuracy) for Repetitive Problem Restructuring in Three Analogical Tasks

\begin{tabular}{|c|c|c|c|c|c|}
\hline & \multicolumn{5}{|c|}{ Restructuring Transformations } \\
\hline & \multicolumn{2}{|c|}{ No Perceptual } & \multicolumn{2}{|c|}{ Perceptual } & \multirow[b]{2}{*}{ Mean } \\
\hline & $\begin{array}{c}\text { No } \\
\text { Semantic } \\
A: B:: C:\end{array}$ & $\begin{array}{l}\text { Semantic } \\
\mathrm{A}: \mathrm{C}: \mathrm{B}:\end{array}$ & $\begin{array}{c}\text { No } \\
\text { Semantic } \\
\text { _:C::B:A }\end{array}$ & $\begin{array}{l}\text { Semantic } \\
\_: B:: C: A\end{array}$ & \\
\hline & \multicolumn{5}{|c|}{ Intact Analogy Repetition } \\
\hline $\begin{array}{l}1 \\
2 \\
3\end{array}$ & $\begin{array}{l}5097 \\
2863 \\
2028\end{array}$ & $\begin{array}{l}5403 \\
3239 \\
2147\end{array}$ & $\begin{array}{l}5223 \\
3401 \\
2093\end{array}$ & $\begin{array}{l}5796 \\
3546 \\
2463\end{array}$ & $\begin{array}{l}5380 \\
3262 \\
2183\end{array}$ \\
\hline \multirow[t]{2}{*}{ Mean } & 3329 & 3596 & 3572 & 3935 & \\
\hline & \multicolumn{5}{|c|}{ Analogy Stem Repetition } \\
\hline $\begin{array}{l}1 \\
2 \\
3\end{array}$ & $\begin{array}{l}4605 \\
2742 \\
1894\end{array}$ & $\begin{array}{l}4838 \\
2867 \\
2246\end{array}$ & $\begin{array}{l}4911 \\
3106 \\
2097\end{array}$ & $\begin{array}{l}5223 \\
3272 \\
2150\end{array}$ & $\begin{array}{l}4894 \\
2997 \\
2097\end{array}$ \\
\hline \multirow[t]{2}{*}{ Mean } & 3080 & 3317 & 3371 & 3549 & \\
\hline & \multicolumn{5}{|c|}{ Analogy Solution Repetition } \\
\hline $\begin{array}{l}1 \\
2 \\
3\end{array}$ & $\begin{array}{r}1874 \\
1019 \\
684\end{array}$ & $\begin{array}{r}2221 \\
1254 \\
835\end{array}$ & $\begin{array}{r}1964 \\
1102 \\
766\end{array}$ & $\begin{array}{r}2042 \\
1166 \\
828\end{array}$ & $\begin{array}{r}2025 \\
1135 \\
778\end{array}$ \\
\hline Mean & 1192 & 1437 & 1277 & 1345 & \\
\hline
\end{tabular}

Table 2

Accuracy Means for Repetitive Problem Restructuring in Three Analogical Tasks

\begin{tabular}{|c|c|c|c|c|c|}
\hline & \multicolumn{5}{|c|}{ Required Restructuring Processes } \\
\hline & \multicolumn{2}{|c|}{ No Perceptual } & \multicolumn{2}{|c|}{ Perceptual } & \multirow[b]{2}{*}{ Mean } \\
\hline & \begin{tabular}{l}
\multicolumn{1}{c}{ No } \\
Semantic \\
$A: B:: C:-$
\end{tabular} & $\begin{array}{l}\text { Semantic } \\
A: C:: B:\end{array}$ & $\begin{array}{c}\text { No } \\
\text { Semantic } \\
\text { _:C::B:A }\end{array}$ & $\begin{array}{l}\text { Semantic } \\
\text { _:C::B:A }\end{array}$ & \\
\hline & \multicolumn{5}{|c|}{ Intact Analogy Repetition } \\
\hline $\begin{array}{l}1 \\
2 \\
3\end{array}$ & $\begin{array}{l}3.24 \\
3.95 \\
5.06\end{array}$ & $\begin{array}{l}2.32 \\
2.41 \\
3.17\end{array}$ & $\begin{array}{l}3.10 \\
4.16 \\
4.05\end{array}$ & $\begin{array}{l}3.03 \\
4.09 \\
4.23\end{array}$ & $\begin{array}{l}2.92 \\
3.65 \\
4.13\end{array}$ \\
\hline \multirow[t]{2}{*}{ Mean } & 4.08 & 2.63 & 3.77 & 3.78 & \\
\hline & \multicolumn{5}{|c|}{ Analogy Stem and Solution Repetition } \\
\hline $\begin{array}{l}1 \\
2 \\
3\end{array}$ & $\begin{array}{l}3.32 \\
4.60 \\
5.63\end{array}$ & $\begin{array}{l}2.63 \\
2.95 \\
3.81\end{array}$ & $\begin{array}{l}3.25 \\
4.29 \\
4.42\end{array}$ & $\begin{array}{l}2.85 \\
3.47 \\
3.82\end{array}$ & $\begin{array}{l}3.01 \\
3.83 \\
4.42\end{array}$ \\
\hline Mean & 4.52 & 3.13 & 3.99 & 3.38 & \\
\hline
\end{tabular}

Note-Accuracy measure $=\operatorname{Ln}[P($ correct $) / P($ error $)]$. 
Intact analogy task. Semantic and perceptual analogy restructuring effects were obtained for response latency in the intact analogy task. The significant $F$ ratios were semantic, $F(1,22)=13.49(\mathrm{p}<.001)$, and perceptual, $F(1,22)=17.12 \quad(p<.001)$. Furthermore, repetition of the intact analogy task significantly decreased response latency $[F(2,45)=217.67, p<.001]$. There were no significant interactions.

For response accuracy, semantic and perceptual analogy restructuring significantly interacted $[F(1,23)=$ $5.00, p<.035]$. The need to perceptually restructure an analogy attenuated the decremental effects of semantic ill-structure for solution latency. Perceptual analogy restructuring did not affect accuracy. Semantic analogy restructuring yielded a marginal main effect for accuracy $[F(1,23)=3.63, p<.069]$. Since failure to semantically restructure was predicted to decrease accuracy, this effect can be interpreted as a significant one-tailed t test $[t(1,23)=1.91, p<.05]$. Repetition, as expected, significantly increased accuracy $[F(2,46)=8.37, p<.001]$.

Analogy stem task. A perceptual restructuring effect was obtained for response latency $[F(1,22)=8.22$, $\mathrm{p}<.009$ ]. Semantic restructuring, however, manifested a marginal effect $[F(1,22)=3.89, p<.061]$. Again, a directional hypothesis was predicted, and this effect was interpreted as a significant one-tailed $t$ test $[t(1,22)=$ $1.97, \mathrm{p}<.05]$. Repetition of the stem task significantly decreased response latency $[F(2,45)=46.47, p<.001]$. A marginal Repetition by Perceptual Restructuring interaction $[F(2,45)=2.94, p<.064]$ indicated that repetition attenuated the perceptual restructuring latency. The least significant difference for the interaction was computed $(268 \mathrm{msec} ; \mathrm{p}<.05)$. The latencies attributable to perceptual restructuring processes for the three repetitions of the analogy stem task were 347 , 384 , and $53 \mathrm{msec}$, respectively. Thus, significant latency differences were obtained for perceptual analogy restructuring in the first two repetitions of the analogy stem task, but not in the third. Accuracy was not assessed in the stem task.

Analogy solution task. A semantic restructuring effect was obtained for response latency in the solution task $[\mathrm{F}(1,22)=5.28, \mathrm{p}<.031]$. Repetition again significantly decreased response latency $[F(2,45)=$ $61.56, \mathrm{p}<.001]$. Repetition significantly increased accuracy for the decomposed analogy tasks $[F(2,46)=$ $14.22, \mathrm{p}<.0011$. The requirement to semantically restructure an ill-structured analogy decreased accuracy $[\mathrm{F}(1,23)=5.61, \mathrm{p}<.027]$.

All other effects produced effect probabilities greater than .10. The response latencies for the intact analogy versions requiring a single but different restructuring process (i.e., the $\mathrm{A}: \mathrm{C}: \mathrm{B}: \mathrm{I}_{-}$and the $-: \mathrm{C}:: \mathrm{B}: \mathrm{A}$ analogies) were not significantly different. Furthermore, the majority of subjects remarked that they were solving the same analogies repeatedly. Often the remarks com- mented on missing an analogy repeatedly or missing one that had previously been solved correctly.

\section{Discussion}

The data supported problem restructuring processes in verbal analogies. Analogies requiring either type of problem restructuring transformations took longer to solve than analogies not requiring that type of problem restructuring. In addition, the two restructuring processes were independent in their effects on response time.

Further, the two processes were executed at different times in the sequence of solution steps. For the decomposed task, perceptual restructuring affected response time during the stem task but had no effect in the solution task. This supported the contention that perceptual restructuring was probably executed at analogy encoding time and completed before the application processes began. Semantic analogy restructuring significantly affected response time in both the analogy stem and solution tasks. Thus, semantic restructuring processes were apparently evoked by the initial relationship and generating processes as well as during the application processes in the solution task.

Accuracy at problem solving was also different for the two restructuring processes in the intact analogy task. Perceptual restructuring processes had no effect on accuracy, whereas semantic restructuring decreased solution accuracy. Most interesting, however, was the interaction of the two analogy restructuring processes for the intact analogy task. The requirement to both perceptually and semantically restructure an analogy attenuated the decremental effect of semantic illstructure. Prior perceptual restructuring of an analogy stem probably facilitates the recognition of semantic ill-structure. Perhaps the added stem processing time involved in perceptual restructuring enabled more exhaustive concurrent relationship eduction or increased the problem solver's sensitivity to analogy ill-structure.

The failure to obtain either a significant Repetition by Perceptual Restructuring interaction in the intact and analogy solution tasks or a Repetition by Semantic Analogy Restructuring interaction in the three analogical tasks for response time was indicative of the automaticity of analogy restructuring. It appears that ill-structured analogies were restructured prior to problem solving, even though subjects knew they had repeatedly solved the same analogy in the same analogy structure. Interestingly, subjects did not adopt the simpler solution recognition strategy for the intact analogies. Thus, both analogy restructuring processes were automatically executed upon perception of ill-structure. It should be noted that this interpretation is based on the failure to reject the null hypothesis.

Two results from the current study suggest that increased repetition might facilitate problem solving and thus attenuate both analogy restructuring latencies. 
First, there was a marginal Perceptual Restructuring by Repetition interaction for response time in the analogy stem task. Second, the latencies attributable to both analogy restructuring processes decreased descriptively with repetition in the intact and analogy stem tasks. While the present results clearly indicate that a solution recognition strategy was not adopted, increasing the number of repetition trials may facilitate a Repetition by Analogy Restructuring interaction. Furthermore, the nature of repetition can be changed so that analogy structure and repetition of problem solving are completely crossed within each subject. These two manipulations may increase the likelihood of obtaining significant Analogy Restructuring by Repetition of Analogy Solving interactions, if they exist. Experiment 2 was performed to test these speculations.

\section{EXPERIMENT 2}

The automaticity of both problem restructuring processes for solving ill-structured verbal analogies was further examined in the second experiment. As in Experiment 1, ill-structured analogies were thought to be restructured prior to problem solving. Both analogy restructuring transformations were expected to be independent and to increase solution latency. The purpose of the first experiment was to obtain support for semantic and perceptual analogy restructuring processes and to determine their stages of execution. The purpose of Experiment 2 was to replicate the first experiment and to obtain a clear examination of the Repetition of Analogy Solving by Analogy Structure interaction. In the first experiment, repetition of problem solving and analogy structure were independent across analogies by counterbalancing in the full data set. However, analogy structure was not varied for each specific analogy that was presented. Rather, each analogy was repeatedly solved in the same analogy structure by the same solver. In Experiment 2, the same four versions of analogy structure were employed. However, each subject solved each analogy four times, once in each of its four structures. While the analogy terms and relationships were repeated, the structure of the analogy was not repeated. Thus, variance attributable to item differences was counterbalanced for each subject. All analogies were solved as intact analogies.

As in Experiment 1, repetition of analogy solving was expected to facilitate analogy solving by making the solution process more algorithmic. Thus, the number of repetitions was increased to four and the type of repetition was changed in an attempt to obtain unconfounded and significant Repetition by Perceptual Restructuring and Repetition by Semantic Restructuring interactions. If significance were obtained, then conclusions about the automaticity of both analogy restructuring processes would not be based on null hypothesis results. Given the results of the first experiment, repetition was expected to attenuate but not eliminate latencies attributable to both types of problem restructuring.

\section{Method}

Subjects and Apparatus. Thirty-two subjects from a large state university in the Midwest participated in the experiment as a requirement of an introductory psychology course. The same reaction time apparatus was used. Each subject solved 48 experimental analogies. This corresponded to 12 replications of each problem structure. The 48 analogies were a subset of the analogies in the first experiment. In addition, the more difficult analogies in the first experiment were not used in the second experiment because solution latency, not accuracy, was of primary interest.

Design and Procedure. The experimental design was a 2 (perceptual structure) by 2 (semantic structure) by 4 (repetition of analogy solving) within-subjects factorial. As in Experiment 1, ANCOVA was used, with response latency as the dependent measure and accuracy as the covariate. Accuracy was also analyzed in a separate univariate case. The four versions of the 48 analogies were put in four random orders, so that each problem version was in a different ordered set. The order of presentation for the four sets was counterbalanced across subjects. The instructions, response options, and procedure were the same as described for the first experiment.

\section{Results and Discussion}

Geometric means and a log accuracy measure were again calculated to estimate solution latency and accuracy, respectively, from the 12 replications. The response latency and accuracy estimates were the units of analysis. Solution latency and accuracy yielded a slight but significant speed-accuracy tradeoff $(r=-.13$, $\mathrm{p}<.001 ; \mathrm{n}=640$ ). Tables 3 and 4 present the adjusted latency means and the accuracy means. The average percent correct was $85 \%$.

Repetition significantly decreased response latency $[F(3,92)=106.10, p<.001]$ and significantly increased accuracy $[F(3,93)=5.12, p<.003]$. Perceptual restructuring increased response latency $[F(1,30)=31.98$, $p<.001]$. Semantic restructuring also increased response latency $[\mathrm{F}(1,30)=29.25, \mathrm{p}<.001]$.

Both the Perceptual Analogy Restructuring by Repetition and the Semantic Analogy Restructuring by Repetition interactions were significant for response latency. The Fratios were Perceptual by Repetition,

Table 3

Solution Latency Means (in Milliseconds Adjusted for Accuracy) for Repetitive Analogy Problem Solving

\begin{tabular}{|c|c|c|c|c|c|}
\hline \multirow[b]{3}{*}{$\begin{array}{c}\text { Repeti- } \\
\text { tions }\end{array}$} & \multicolumn{5}{|c|}{ Required Restructuring Processes } \\
\hline & \multicolumn{2}{|c|}{ No Perceptual } & \multicolumn{2}{|c|}{ Perceptual } & \multirow[b]{2}{*}{ Mean } \\
\hline & $\begin{array}{l}\quad \text { No } \\
\text { Semantic } \\
\text { A:B::C:_ }\end{array}$ & $\begin{array}{l}\text { Semantic } \\
\text { A:C::B: }\end{array}$ & $\begin{array}{c}\text { No } \\
\text { Semantic } \\
\text { _:C::B:A }\end{array}$ & $\begin{array}{r}\text { Semantic } \\
\text { _:B::C:A }\end{array}$ & \\
\hline 1 & 4661 & 5529 & 5594 & 6156 & 5485 \\
\hline 2 & 3620 & 3891 & 3929 & 4223 & 3916 \\
\hline 3 & 2822 & 3078 & 2985 & 3263 & 3037 \\
\hline 4 & 2312 & 2584 & 2576 & 2772 & 2561 \\
\hline Mean & 3354 & 3770 & 3771 & 4104 & \\
\hline
\end{tabular}


Table 4

Solution Accuracy Means for Repetitive Analogy Problem Solving

\begin{tabular}{|c|c|c|c|c|c|}
\hline \multirow[b]{3}{*}{$\begin{array}{l}\text { Repeti- } \\
\text { tions }\end{array}$} & \multicolumn{5}{|c|}{ Required Restructuring Processes } \\
\hline & \multicolumn{2}{|c|}{ No Perceptual } & \multicolumn{2}{|c|}{ Perceptual } & \multirow[b]{2}{*}{ Mean } \\
\hline & \begin{tabular}{l}
\multicolumn{1}{c}{ No } \\
Semantic \\
$\mathrm{A}: \mathrm{B}: \mathrm{C}:-$
\end{tabular} & $\begin{array}{l}\text { Semantic } \\
A: C:: B:\end{array}$ & $\begin{array}{c}\text { No } \\
\text { Semantic } \\
\text { _:C::B:A }\end{array}$ & $\begin{array}{c}\text { Semantic } \\
\text { _:B::C:A}\end{array}$ & \\
\hline 1 & 3.02 & 2.07 & 2.69 & 2.06 & 2.46 \\
\hline 2 & 2.70 & 3.28 & 2.51 & 2.20 & 2.67 \\
\hline 3 & 2.27 & 2.76 & 3.20 & 3.15 & 2.85 \\
\hline 4 & 3.27 & 3.36 & 3.08 & 3.35 & 3.27 \\
\hline Mean & 2.82 & 2.87 & 2.87 & 2.69 & \\
\hline
\end{tabular}

Note-Accuracy measure $=L n / P($ correct $) / P($ error $)]$.

$F(3,92)=8.26(p<.001)$, and Semantic by Repetition, $F(3,92)=3.36(p<.022)$. The two ill-structured analogies requiring a single restructuring transformation produced nearly identical response latency functions. For both interactions, the latency attributable to analogy restructuring diminished with repetition. Simple main effects tests for the first and last repetition intervals were computed. Both effects of problem restructuring were significant in both repetition intervals. At the first level of repetition, the $F$ ratios were perceptual, $F(1,30)$ $=44.73(\mathrm{p}<.001)$, and semantic, $F(1,30)=9.98$ $(p<.004)$. At the fourth level of repetition, the $F$ ratios were perceptual, $F(1,30)=4.68(p<.039)$, and semantic, $F(1,30)=12.76(\mathrm{p}<.001)$.

As is evident in Table 3, the greatest facilitation of repetition was between the first and second intervals. Roughly, the amount of facilitation due to repetition halved with each additional solution. The constantly diminishing change in slope is indicative of a uniform facilitation effect, as opposed to a sudden strategy shift. Thus, repetitive analogy solving attenuated but did not eliminate analogy restructuring processes. No other factors were significant (all ps $>.10$ ).

As in Experiment 1, the independence of perceptual and semantic analogy restructuring was determined. Thus, the two stages are additive portions of the overall solution time (Pachella, 1974). Figure 1 illustrates the additive nature of the analogy restructuring processes. Notice that the $-: C:: B: A$ and the $A: C:: B:{ }_{-}$means are nearly identical. Perhaps the two restructuring processes share similar elementary information processes (Newell \& Simon, 1972) that are used to restructure the problem space and thus have equivalent durations.

\section{GENERAL DISCUSSION}

Although the type of problem repetition varied across the two experiments, there were three main and consistent results. First, both experiments supported the contention that ill-structured verbal analogies are restructured to a well-structured form in the course of problem solving. Ill-structured analogies requiring either perceptual or semantic analogy restructuring were solved slower than well-structured analogies that did not require analogy restructuring.

Second, two qualitatively distinct analogy restructuring stages were evoked by ill-structured analogies. The two restructuring processes were independent and differed on their accuracy and stage of execution. Latencies for perceptual and semantic analogy restructuring processes did not interact in either experiment. In addition, semantically ill-structured analogies had higher error rates than semantically well-structured analogies in the first experiment, whereas perceptually ill-structured analogies were solved as accurately as perceptually well-structured analogies in both experiments. Furthermore, the structure of the analogy was supported as influencing the execution sequence for problem restructuring and problem solving processes. In Experiment 1, significant latencies for perceptually restructuring an analogy were observable for the intact analogy and analogy stem tasks, but not for the analogy solution task. Presumably, perceptual restructuring processes were triggered by the position of the blank term in the stem. Significant latencies for semantic analogy restructuring processes were obtained in all three analogical tasks. Thus, semantic analogy ill-structure was perceived during stem representation and, more

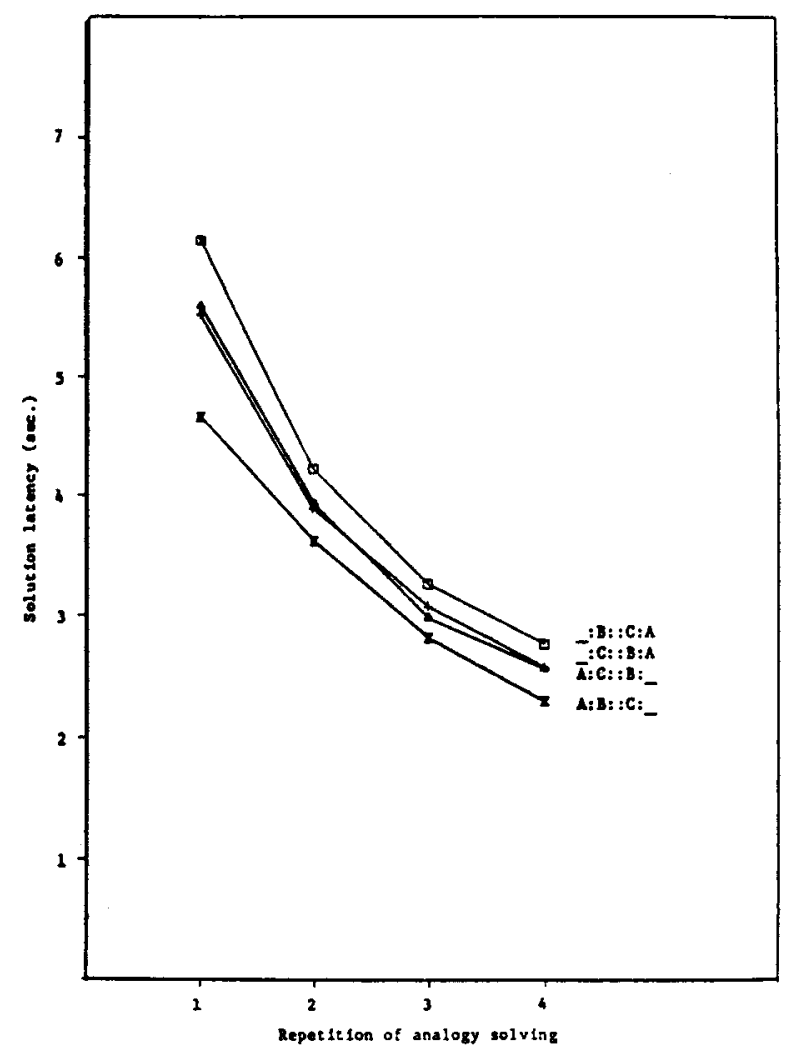

Figure 1. Repetitive solution latencies for well- and illstructured verbal analogies. 
likely, during and following alternative evaluation. While perceptual restructuring processes probably have a fixed trigger and are executed at the beginning of stem processing, semantic ill-structure is less rapidly and accurately perceived and semantic restructuring processes can be executed throughout the course of analogy solving.

Third, analogy restructuring exhibited automaticity as the relationships in the problem solving space became reproductive and the solution steps became more algorithmic. Repetition of analogy solving significantly reduced problem representation and solution time and increased accuracy for both experiments. Furthermore, repetition did not eliminate analogy restructuring processes in either experiment. In the second experiment, perceptual and semantic restructuring processes interacted with repetition of analogy solving. This interaction illustrated that the latency attributable to both analogy restructuring processes decreased with repetitive analogy solving but was not eliminated. Thus, the execution of analogy restructuring processes was facilitated. Presumably, an algorithm for solving ill-structured analogies was developed and refined with repetition of analogy solving.

The results of both experiments indicate that current information processing models of analogical reasoning should be extended to incorporate both analogy restructuring processes for ill-structured analogies. Additionally, the restructuring processes and the other components of analogical reasoning may be fairly automatic. The remainder of the discussion will address these two issues.

\section{Problem Restructuring Processes and Models}

As mentioned previously, current models of analogical reasoning have not addressed the issue of problem restructuring. For current models of analogical reasoning to be capable of problem restructuring, they must be extended to include restructuring components. Additionally, the sequence and nature of component execution must be specified.

The proposed analogy restructuring extensions are applicable to current models of analogical reasoning. In the extended model, problem restructuring processes are evoked only for ill-structured analogies. Thus, both restructuring processes are optional and are performed only when ill-structure is perceived. Perceptual restructuring during encoding is evoked by the presence of the blank stem term. Semantic restructuring processes are primarily evoked when the initial relationships of the analogy's domain are not applicable to the alternatives. Semantic restructuring processes could also be evoked after inference. This would occur when the $A: C$ pair is strongly related and the A:B pair is not related or is less strongly related.

In such an extended model, the $\_: C:: B: A$ and the _:B::C:A analogies are perceptually restructured during encoding of the stem. The $\mathrm{A}: \mathrm{B}: \mathrm{C}:$, and the $\mathrm{A}: \mathrm{C}: \mathrm{B}:$ :_ $_{-}$analogies are encoded as perceptually well-structured analogies. The $A: C:: B:$, and the _.:B::C:A analogies are semantically restructured either during or, less frequently, prior to application. The $\mathrm{A}: \mathrm{B}: \mathrm{C}:$, $_{-}$and the $-: \mathrm{C}: \mathrm{B}: \mathrm{A}$ analogies are not semantically restructured. Thus, the extended model of analogical reasoning requires perceptual and semantic restructuring for various ill-structured analogies. Further research is needed to resolve issues about process execution sequences, especially for other ill-structured versions of analogies and for more difficult analogies. One criticism of experimental work on analogical reasoning is that only easy analogies have been investigated (Pellegrino \& Lyons, 1979). Nevertheless, it is apparent that easy ill-structured analogies are restructured and that the sequence of solution steps differs with the type of initial analogy structure.

Furthermore, the results of Experiment 1 tentatively indicate that the incorporation of semantic analogy restructuring components might increase the fit between models of analogical reasoning and individual differences for analogies on aptitude tests, since psychometric analogies are often ill-structured. Apprehension of problem ill-structure and the ability to restructure ill-structured problems may be important information processing abilities in regards to the linkage of information processing models of cognition, theories of intelligence, and intelligence assessment.

\section{Automaticity of Analogical Reasoning}

In the first experiment, three repetitions of verbal analogies failed to eliminate the effects for either analogy restructuring process for intact analogies. A solution recognition strategy was not adopted, even though subjects knew they had solved the same analogy twice before. Furthermore, repetition reduced response latency and increased accuracy in both experiments. In the second experiment, each analogy was solved four times, each in a different problem structure. In this way, the analogy's relationships, not its structure, were repeated. In the second experiment, as in the first, repetition attenuated but did not eliminate analogy restructuring effects.

Because the nature of repetition varied across both experiments, the repetition effects are not directly comparable. However, the shape of the obtained facilitation functions supports the contention that the analogy restructuring and the analogical processes executed after analogy restructuring were automatically executed. Otherwise, a strategy shift to solution recognition would have produced a marked flattening of the repetition function for intact analogies in both experiments.

It is not surprising to obtain automaticity of problem restructuring for verbal analogies. Recall that verbal analogy problems tap basic and pervasive components of thinking. These components are the eduction, pro- 
duction, and evaluation of relationships among word concepts. These semantic components and vocabulary are probably the reasons verbal analogies saturate general factors of intelligence. Spearman (1923, pp. 63 and 91) was the first to comment on the possible automaticity of verbal reasoning when he speculated on the nature of relationship eduction processes. He described two cognitive processes, the eduction of relations (inference) and the eduction of correlates (application), as operations that produced the "immediate knowing" of relations. Additionally, the observation that subjects cannot easily explain how they know relationships and solve analogies suggests that analogical reasoning is probably automatic. The current results suggest that analogy restructuring is not susceptible to strategic control and thus is automatic, at least for verbal analogies at this level of difficulty.

Algorithmic analogical reasoning. Assuming that analogical reasoning is fairly automatic, what produced the repetition effects? Since facilitation cannot be attributed to the absence of problem solving processes, the processes utilized must, to differing degrees, be individually facilitated. Such facilitation could be attributable to memory retrieval and learning effects. Memory retrieval processes are evoked by many analogical processes. Assuming a spreading activation memory model (Anderson, 1976; Collins \& Loftus, 1975) or other strength memory model, repetitive information retrieval would be facilitated and thus expected to decrease solution time.

Learning effects would also be expected with repetition of the constituent problem solving processes. An algorithm for analogy solution could be learned. For example, the execution of a specific inference, or analogy restructuring process, at a specific step in the solution process would become "known" and its execution facilitated because it had been done several times before. When elementary information processes are modeled as production systems, as in ACT (Anderson, 1976), repetition can facilitate the selection and execution of productions. In a similar manner, specific execution sequences for analogy solving processes could become learned and performed faster, given repetitive problem solving. Thus, repetition could make analogical reasoning more algorithmic by facilitating memory retrieval and by facilitating the selection and execution of analogical processes.

\section{Conclusions}

Both experiments supported the hypotheses that perceptually and semantically ill-structured analogies were restructured prior to problem solving. Main effects for two independent analogy restructuring processes were obtained. Furthermore, the two analogy restructuring processes were executed at different stages of problem solving. Repetition of problem solving did not eliminate analogy restructuring, although it did attenu- ate the effects of analogy structure. Thus both analogy restructuring processes were concluded to be fairly automatic. Repetition was thought to attenuate the effects of analogy structure by making successive problem solving more algorithmic and by facilitating memory retrieval. A perceptual and a semantic restructuring process were proposed as extension to current models of analogical reasoning.

\section{REFERENCE NOTE}

1. Barnes, G. M. A concurrent model for solving well- and illstructured analogies. Unpublished doctoral thesis, University of Kansas, May 1980.

\section{REFERENCES}

Anderson, J. R. Language, memory, and thought. Hillsdale, N.J: Erlbaum, 1976.

Clark, H. H., \& Chase, W. G. On the process of comparing sentences against pictures. Cognitive Psycshology, 1972, 3, 472-512.

Collins, A. M., \& Loftus, E. J. Spreading activation theory of semantic information-processing. Psychological Review, 1975, 82, 407-428.

Davis, G. A. Psychology of problem solving: Theory and practice. New York: Basic Books, 1973.

Duncken, K. On problem solving. Psychological Monographs, 1945, 58(Whole No. 270).

Evans, T. G. A program for the solution of geometric-analogy intelligence test questions. In M. Minsky (Ed.), Semantic information processing. Cambridge, Mass: M.I.T. Press, 1968.

Greeno, J. G. The structure of memory and the process of solving problems. In R. L. Solso (Ed.), Contemporary issues in cognitive psychology: The Loyola symposium. Washington D. C.: Winston, 1973.

Greeno, J. G. Indefinite goals in well structured problems. Psychological Review, 1976, 83, 479-491.

GRUDIN, J. Processes in verbal analogy solution. Journal of Experimental Psychology: Human Perception and Performance, $1980,6,67-74$.

Hayes-Roth, F., \& McDermott, J. An interference matching technique for inducing abstractions. Communications of the Association of Computing Machinery, 1978, 21, 401-410.

Johnson, D. M. Serial analysis of verbal analogy problems. Journal of Educational Psychology, 1962, 53, 86-88.

Luchins, A. S. Mechanization in problem solving. Psychological Monographs, 1942, 54(Whole No. 248).

Maltzman, I. Thinking: From a behaviorist point of view. Psychological Review, 1955, 62, 275-280.

McNemar, Q. Lost: Our intelligence? Why? American Psychologist, 1964, 19, 871-882.

MEDNICK, S. A. The associative basis of the creative process. Psychological Review, 1962, 69, 220-230.

Newelt, A., \& Simon, H. A. Human problem solving. Englewood Cliffs, N.J: Prentice Hall, 1972.

Pachella, R. G. The interpretation of reaction-time in information processing research. In Kantowitz, B. H. (Ed.), Human information-processing: Tutorials in performance and cognition. Hillsdale, N.J: Erlbaum, 1974.

Pellegrino, J. W., \& Glasser, R. Components of inductive reasoning. In R. E. Snow, P. A. Frederico, \& W. Montague (Eds.), Aptitude, learning, and instruction: Cognitive process analysis. Hillsdale, N.J: Erlbaum, 1980.

Pellegrino, J. W., \& Lyon, D. R. The components of a componential analysis. Intelligence, 1979, 3, 169-186. 
Potтs, G. R., \& Scholz, K. W. The internal representation of a three term series problem. Journal of Verbal Learning and Verbal Behavior, 1975, 14, 471-480.

Reitman, W. Cognition and thought. New York: Wiley, 1965.

Rumelhart, D. E., \& Abrahamson, A. A. A model for analogical reasoning. Cognitive Psychology, 1973, 5, 1-28.

Simon, H. A. The structure of ill structured problems. Artificial Intelligence, 1973, 4, 181-201.

Simon, H. A., \& REED, S. K. Modeling strategy shifts in a problem solving task. Cognitive Psychology, 1976, 8, 86-97.

SPEARMAN, C. The nature of intelligence and principles of cognition. London: Macmillan, 1923.

SternberG, R. J. Component processes in analogical reasoning. Psychological Review, 1977, 34, 353-378. (a)

STE RNBERG, R. J. Intelligence, information-processing and analogical reasoning: The componential analysis of human abilities. Hillsdale, N.J: Erlbaum, 1977. (b)
Sternberg, R. J. The nature of mental abilities. American Psychologist, 1979, 34, 214-230.

Whitely, S. E. Relationships in analogies: A semantic component of a psychometric task. Educational and Psychological Measurement, 1977, 37, 725-739.

Whitely, S. E., \& Barnes, G. M. The implications of processing event sequences for theories of analogical reasoning. Memory \& Cognition, 1979, 7, 323-331.

Whitely, S. E., \& DAwis, R. V. The influence of test context on item difficulty. Educational and Psychological Measurement, 1976, 36, 329-337.

(Received for publication June 23, 1980; revision accepted December 16, 1980.) 